\title{
Is there a Relation between ABO Blood Group and Malaria?
}

\author{
Sangita Vasava*, Sucheta Lakhani and Jitendra Lakhani \\ Department of Microbiology and Gen. Medicine SBKS MI \& RC, \\ Piparia, Waghodia-391760, Gujarat, India \\ *Corresponding author
}

\section{Keywords}

ABO blood group,

Malaria, Giemsa

stain

Article Info

Accepted:

10 February 2019

Available Online:

10 March 2019

\section{A B S T R A C T}

Malaria occurrence and its severity have been related to many host factors including $\mathrm{ABO}$ blood groups. This cross sectional study analyzes severity and prevalence of malaria in various $\mathrm{ABO}$ blood groups. This prospective observational study was carried out in 74 peripheral smear positive malaria patients. Blood group analysis was done in these selected patients as well as in 74 control subjects who came for voluntary blood donation. Necessary investigations and Malarial antigen test were done. Patients were clinically examined and grouped into mild, moderate and severe malaria by defined clinical criteria. Cases of P.vivax $(54.05 \%)$ were more than P.falciparum (08.10\%) and mixed infection (P.vivax+P.falciparum) $(37.83 \%)$. The age group which was commonly affected was 18 30 years $(39.18 \%)$. Elderly patients $>60$ years $(05.40 \%)$ were affected least. Prevalence of malaria was higher in males in comparison with females. \{Males (71.62\%) Vs females $(28.37 \%)\}$.Malaria cases in different blood groups were $39.18 \%$ in 'B', $27.2 \%$ in ' $\mathrm{A}$ ', $22.97 \%$, in ' $\mathrm{O}$ ' and $10.81 \%$ in 'AB' blood groups. Prevalence of $\mathrm{ABO}$ blood groups was very similar in controls. Thus we did not find any selection bias of malaria in a particular blood group. Protection in regards to severity was found in ' $\mathrm{O}$ ' blood group patients. No special predilection was noted in regards to blood group and malaria. ' $O$ ' blood group had less severe malaria cases.

\section{Introduction}

In 2016, WHO reported 216 million cases of malaria from 91 countries India is endemic for malaria (WHO, World malaria report, 2016). Present study is conducted at our center which caters services to Gujarat and Madhya Pradesh, having perennial malaria cases and has high load of sickle cell anemia. There are many host factors which gives protection to malaria like Sickle cell disorder, G-6-PD deficiency and ' $\mathrm{O}$ ' blood group. Geographical location of prevalence of malaria and sickle cell disorder was same for both, a fact to be understood for future interventions (Lakhani et al., 2017). Hypothesis about selective genetic pressure from malaria infection on the human population which resulted in several erythrocyte polymorphisms comes from the fact that geographical distribution of these genetic variations and malaria was same. Influence of malaria on blood group variations 
is also one such hypothesis (Christine et al., 2007). Evidence suggests that origin, distribution and relative proportion of $\mathrm{ABO}$ blood groups of today in community may be result of past influence of malarial infection (Singh et al., 2015). Prevalence of malaria is higher in blood group $\mathrm{B}$, while ' $\mathrm{O}$ ' may confer resistance ( Aditya et al.,2011).

$P$ falciparum infection severity is related with cytoadherence due to rosetting of red blood cells inside the capillaries, which in turn is related to $\mathrm{ABO}-$ blood groups. Blood group ' $\mathrm{O}$ ' is found to bestow protection from severe falciparum malaria due to decrease in rosetteeing effect (Rowe et al., 2007).

\section{Materials and Methods}

This observational study was conducted at SBKS Medical Institute and Research Center which is attached to Dhiraj hospital, a multispeciality hospital, situated at village Piparia, Taluka Whaghodia, Dist: Vadodara. Study was conducted to find out association of $\mathrm{ABO}$ blood group to malaria cases. Study was conducted on patients who took services from our institute between June 2015 and December 2015. This study was approved by the institutional ethics committee (IEC). 74 patients having malaria diagnosed by Giemsa stained peripheral smears attending OPD or admitted to indoor wards or critical care wards were recruited in this study as cases.74 controls were randomly selected who came for voluntary blood donation during the study period. Thick and Thin blood smears were prepared using fresh blood sample and stained with Giemsa's stain. Malarial antigen test done for P.falciparum, P.vivax and for mixed infections species identification was reported after examining smear and antigen report combinedly. Parasite count, other hematological and biochemical tests were performed to decide severity of malaria and organ involvement. ABO blood grouping was done by slide method by using antisera-A, antisera-B, antisera-D, method based on principle of agglutination. All OPD patients and post discharge indoor patients were followed up for two weeks. Patients whose peripheral smear were negative for malarial parasites and had clinical malaria were not taken for the study.

Patients were divided in three categories of severity of malaria. Mild variety was patients who were treated at OPD basis and did not require indoor admission or injectable preparations and did not fall in criteria listed in moderate and severe malaria. Moderate grade of malaria were the patients who required indoor admission for observation and treatment. Treatment included injectable antimalarial drugs or injectable treatment for other accompaniments of malaria. Patients of malaria who required ICU admission, had single organ involvement listed in WHO publication of tropical medicine and international health (2014) or having Multiorgan dysfunction syndrome (MODS) were grouped as severe malaria. Patients with hyperparasitemia $(>250,000$ parasites per $\mu l$ of blood) were also consider in severe disease despite not having organ involvement or not needing ICU admission. WHO (2014) criteria of severe malaria includes Impaired consciousness, Acute Respiratory distress, Acidotic breathing, Multiple convulsions, Prostration, Abnormal bleeding, Jaundice, Severe anaemia on lab. Investigation, Hypoglycaemia, Metabolic acidosis on ABG finding, Acute Kidney Injury and Hyperparasitaemia.

\section{Results and Discussion}

Out of 74 cases, 40 had $P$. vivax (54.05\%),06 had P.falciparum (08.10\%) and 28 had mixed infection (P.vivax + P.falciparum) (37.83\%) (Table 1). As table 2 shows there were 19 patients in age group below 18 years, 
maximum patients i.e. 29 in age group of 18 to 30 years, 22 in age group of 31 to 60 years and 04 patients above 60 years. Prevalence of malaria was higher in males in comparison with females. \{Males (71.62\%) Vs females (28.37) (Table 3).

Out of 74 malaria cases, $20(27.02 \%)$ had ' $A$ ' blood group of which 18 were Rh positive and 2 were negative. 29 (39.18\%) had ' B' blood group (28 B positive,01 negative), 17 (22.97\%) had 'O' blood group (16 ' $\mathrm{O}$ ' positive,01 negative) and $08(10.81 \%)$ had ' $A B$ ' positive' blood group (Table 4). Malaria cases were more in 'B' blood group (39.18\%) and least affected was 'AB' blood group (10.81\%). When it was compared to control group, frequency of diff blood groups were same in selected malaria pat and control group. For comparison purpose malaria cases were distributed in group I and II of which group II consisted of 40 cases of vivax malaria only, Group I patients consisted of 6 falciparum malaria cases and 28 mixed infections having falciparum and vivax infection combined. Group I had total 34 patients. As table 5 shows, Out of 17 cases of blood group 'O', 10 were having vivax malaria while 7 had falciparum malaria and mixed infection. 'AB' blood group which was present in 8 malaria cases, 1 had vivax while rest 7 were having falciparum and mixed infection. When Fisher Exact test was applied to compare ' $\mathrm{O}$ ' blood group vs other blood groups in relation to vivax and falciparum and/or mixed infection (I and II group) no significant difference was observed (Table 5).

Table 6 shows distribution of ABO Blood group in relation to severity of malaria. $28(37.83 \%)$ were grouped in mild malaria, $24(32.43 \%)$ were in moderate malaria and 22 $(29.72 \%)$ were having severe malaria. Of 17 Blood group 'O' malaria cases, 10 were having mild malaria while 05 had moderate and 02 had severe malaria. When Fisher Exact
Test was applied to compare mild and severe malaria in ' $\mathrm{O}$ ' blood group patient in comparison to 'A', 'B', and 'AB' blood groups, statistically significant difference was found $(\mathrm{P}<0.05)$. Other blood groups having severe malaria were $08(36.36 \%), 10(45.45 \%)$ and $02(09.09 \%)$ in blood group 'A', 'B', and 'AB' respectively. Prevalence of severe malaria was more in ' $\mathrm{B}$ ' blood group however when compared mild and severe malaria in relation to ' $\mathrm{B}$ ' blood group and rest, no significant difference was obtained. Moderate malaria was in $24(32.43 \%)$ cases of which $07(29.16 \%)$ cases were present in ' $\mathrm{A}$ ' and ' $\mathrm{B}$ ' blood group while $05(20.83 \%)$ cases were in ' $\mathrm{O}$ ' and ' $\mathrm{AB}$ ' blood group. Mild malaria in blood group 'A', 'B', 'O' and 'AB' were $05(17.85 \%), \quad 12(42.85 \%), \quad 10(35.71 \%)$ and $01(03.57 \%)$ respectively.

Malaria is one of the diseases known since ancient time. It has lead to heavy burden on human health. Malarial parasite is affecting red blood cells and thus changes in red cell milieu may change susceptibility, resistance, or severity of malarial infection. Modification of hemoglobin structure, alteration in cytosolic enzyme Glucose-6- phosphate dehydrogenase (G6PD or G6PDH) quantity and variation in $\mathrm{ABO}$ blood groups antigens is presumed to be result of malaria. Erythrocytic polymorphism leading to occurrence of several different forms of blood groups types in humans and its relation to malaria is worth investigation. Present study was undertaken to know whether $\mathrm{ABO}$ blood group had any relation to malaria or not. Available literature is more related with falciparum malaria however present study included vivax and mixed malarial infection also.

There are number of studies available in literature in regards to various epidemiological and host factors which consist of genetic blood polymorphisms including $\mathrm{ABO}$ blood groups and their relation to protection 
hypothesis in malaria. Results of these studies are varied and non consistent. It may be because of changes in environment and also due to altered epidemiology of malaria at present time. Migration of native population to other places, marriages between different races and because of many more reasons, the results of different study may not reflect same results. Apart from host related factors, agent related factors like inclusion of the cases of different species of malarial parasites, different criteria in regards to severity of malaria and others had resulted into heterogenicity of the results. In Our study we did not find any prevalence difference of malaria in different blood groups. It may be because of small sample size of our study patients. Different studies are available which gives conclusion that malaria is more prevalent in ' $B$ ' blood group while other studies conclude that it is more prevalent in 'A' blood group. Studies are also available suggesting that malaria is more common in both, 'A' and 'B' blood group. Similary like in our study, Number of studies have proved no relation with blood group.

Thakur et al., (1992) did study on tribal community and compared results with Delhi community subjects. Their indirect conclusion is like our study that ABO blood group does not have relation with malaria prevalence. In the study at Thakur and Verma (1992) ABO blood groups did not differ significantly in tribal and Delhi subjects. Assumption of tribal population to have different blood group than others were not found true in this study. They also did not find difference in malarial antibodies among subjects with different blood groups.

Many studies available in literature are cross sectional studies and are without comparison data with control subjects. Control group will be very important as prevalence of blood groups varies at different geographical locations. Ours Study had prevalence of malaria higher in ' $\mathrm{B}$ ' blood group, however ours control group also had higher prevalence of blood group 'B'.

In our study we had taken control group from voluntary blood group donor and they were of similar number as malaria cases.

Aditya et al., (2011) showed that patients with blood group ' $\mathrm{B}$ ' have four fold increased risk of developing severe infection. Though apparently higher severe malaria cases were present in ' $\mathrm{B}$ ' blood group result was not statistically significant.

Studies which have shown prevalence of malaria in 'B' blood group. In our study ' $B$ ' blood group was present in 29 of 74 patients and 27 of 74 control patients. Of 29 patients 19 had vivax and 10 had either falciparum or mixed infection.10 of 22 severe malaria patients was seen in 'B' group (45.45\%).

As blood group may have geographical and racial variation, we had taken control group also. In our control group which had similar number of patients (74), 'B' blood group was present in 27 (36.48\%), 'A' blood group in $24(34.43 \%)$, 'O' blood group in $19(25.67 \%)$ and 'AB' blood group in 04(05.40\%).Our study did not find relation between prevalence of malaria and blood group. In the similar study most common blood group among the 99 malaria patients was group ' $\mathrm{O}$ ' $(38.38 \%)$ followed by blood groups 'A' $(32.32 \%)$, 'B' $(22.22 \%)$, and ' $A B$ ' $(7.07 \%)$ respectively. The distribution of $\mathrm{ABO}$ blood types among the control group was 'A' (32\%), 'B' (23\%), 'O' $(32 \%)$, and 'AB' $(13 \%)$ respectively (Xuan et al., 2017).

Thirteen genetic blood polymorphisms which included $\mathrm{ABO}$ and other blood group systems as well as enzymes were investigated by (Beiguelman et al., 2003) in Brazil where 
malaria is endemic like in India. Duffy system was found to be associated with susceptibility to malaria. In study of Samuel K et al., (1979), the Duffy blood group antigen $\mathrm{Fy}^{\mathrm{a}}$ and $\mathrm{Fy}^{\mathrm{b}}$ were absent in falciparum infected and control group Nigerian children.

Studies have shown high frequency of malaria episodes among blood group A individuals in Zimbawe (Fisher and Boone et al., 1998). In study by Fischer et al., severe malaria having CNS involvement and coma as well as severe anemia was found associated with blood group
'A'. The study conducted by Gayathri et al., (2013) had total 205 patients of which 123 were of P.falciparum, 78 of P.vivax and 04 had mixed infected. Blood group amongst them were ' $\mathrm{A}$ ' in 33 , ' $\mathrm{B}$ ' in 84, ' $\mathrm{O}$ ' in 70 and ' $A B$ ' in 18. They concluded that blood group ' $A$ ' and ' $\mathrm{B}$ ' are more susceptible to malaria infection as compared to blood group ' $\mathrm{O}$ '.

In our study, 22 patients had severe Malaria, of which 10 were of blood group 'B' $(45.45 \%)$ and only 2 were from blood group ' $\mathrm{O}$ ' $(09.09 \%)$.

Table.1 Distribution of malaria according to species of malarial parasites

\begin{tabular}{|l|l|c|c|}
\hline Group & Parasite & $\begin{array}{c}\text { Number of } \\
\text { Cases }(\mathbf{n}=\mathbf{7 4})\end{array}$ & Percentage of Cases \\
\hline $\mathbf{1}$ & P. falciparum & 06 & 08.10 \\
\hline $\mathbf{2}$ & P. vivax & 40 & 54.05 \\
\hline $\mathbf{3}$ & P.falciparum + P. vivax & 28 & 37.83 \\
\hline & Total & 74 & 100 \\
\hline $\mathbf{A}(\mathbf{1 + 3})$ & P.falciparum+Mixed Infection & 34 & 45.95 \\
\hline $\mathbf{B}(\mathbf{2})$ & P. vivax only & 40 & 54.09 \\
\hline
\end{tabular}

Table.2 Prevalence of malaria by Age

\begin{tabular}{|l|l|l|l|l|l|}
\hline $\begin{array}{l}\text { Age } \\
\text { (years) }\end{array}$ & $\begin{array}{l}P \text {.falciparum } \\
\text { and \% }\end{array}$ & no. & P. vivax no. and \% & $\begin{array}{l}\boldsymbol{P} \text {. falciparum }+. \\
\text { vivax } \text { no. and \% }\end{array}$ & $\begin{array}{l}\text { Total no. } \\
\text { and \% }\end{array}$ \\
\hline $\begin{array}{l}<\mathbf{1 8} \\
\text { years }\end{array}$ & $02(33.33)$ & $09(22.50)$ & $08(28.57)$ & $19(25.67)$ \\
\hline $\mathbf{1 8 - 3 0}$ & $03(50.00)$ & $17(42.50)$ & $09(32.14)$ & $29(39.18)$ \\
\hline $\mathbf{3 1 - 6 0}$ & $01(16.66)$ & $13(32.50)$ & $08(28.57)$ & $22(29.72)$ \\
\hline$>$ >60 & 00 & $01(02.50)$ & $03(10.71)$ & $04(5.40)$ \\
\hline Total & $06(8.11)$ & $40(54.09)$ & $28(37.84)$ & $74(100)$ \\
\hline
\end{tabular}

Table.3 Prevalence of malaria by sex

\begin{tabular}{|l|l|l|l|l|}
\hline Sex & $\begin{array}{l}\boldsymbol{P} \text {.falciparum no. } \\
\text { and \% }\end{array}$ & $\begin{array}{l}\boldsymbol{P} \text {. vivax no. and } \\
\text { \% }\end{array}$ & $\begin{array}{l}\boldsymbol{P} \text {. falciparum } \boldsymbol{+} \text {. } \\
\text { vivax } \text { no. and \% }\end{array}$ & $\begin{array}{l}\text { Total no. } \\
\text { and \% }\end{array}$ \\
\hline Male & $05(83.33)$ & $27(67.50)$ & $21(75.00)$ & $53(71.62)$ \\
\hline Female & $01(16.66)$ & $13(32.50)$ & $07(25.00)$ & $21(28.37)$ \\
\hline Total & 06 & 40 & 28 & $74(100)$ \\
\hline
\end{tabular}


Table.4 Distribution of ABO blood groups in cases as well as in controls

\begin{tabular}{|l|l|l|}
\hline Blood Group & Malaria Cases no. and \% & Controls no. and \% \\
\hline A +ve & $18(24.32)$ & $24(32.43)$ \\
\hline A-ve & $02(02.70)$ & 00 \\
\hline A total & $20(27.02)$ & $24(32.43)$ \\
\hline B+VE & $28(37.83)$ & $27(36.48)$ \\
\hline B-VE & $01(01.35)$ & 00 \\
\hline B total & $29(39.18)$ & $27(36.48)$ \\
\hline O +ve & $16(21.62)$ & $17(22.97)$ \\
\hline O-VE & $01(01.35)$ & $02(02.70)$ \\
\hline O total & $17(22.97)$ & $19(25.67)$ \\
\hline AB +ve & $08(10.81)$ & $04(05.40)$ \\
\hline AB - ve & 00 & 00 \\
\hline AB total & $08(10.81)$ & $04(05.40)$ \\
\hline Total & $74(100)$ & $74(100)$ \\
\hline
\end{tabular}

Table.5 Distribution of ABO blood groups and species of malaria parasites

\begin{tabular}{|l|l|l|l|}
\hline $\begin{array}{l}\text { Blood } \\
\text { group }\end{array}$ & $\begin{array}{l}\text { Group-I (P.falciparum } \\
\text { +mixed infection) no. }(\boldsymbol{\%})\end{array}$ & $\begin{array}{l}\text { Group-II } \\
\text { P.vivax no. }(\%)\end{array}$ & Total no (\%) \\
\hline A & $(1+9) 10(29.41 \%)$ & $10(25 \%)$ & $20(27.02 \%)$ \\
\hline B & $(2+8) 10(29.41 \%)$ & $19(47.5 \%)$ & $29(39.18 \%)$ \\
\hline O & $(1+6) 07(20.58 \%)$ & $10(25 \%)$ & $17(22.97)$ \\
\hline AB & $(2+5) 07(20.58 \%)$ & $01(2.5 \%)$ & $08(10.81)$ \\
\hline Total & $34(100)(45.95 \%)$ & $40(100) 54.05 \%$ & $74(100)$ \\
\hline
\end{tabular}

Table.6 Distribution of ABO Blood group according to severity of malaria

\begin{tabular}{|l|l|l|l|l|}
\hline Blood Group & $\begin{array}{l}\text { Mild } \\
\text { malaria*(n=28) }\end{array}$ & $\begin{array}{l}\text { Moderate } \\
\text { malaria(n=24) }\end{array}$ & $\begin{array}{l}\text { Severe } \\
\text { malaria*(n=22) }\end{array}$ & Total(n=74) \\
\hline A & $05(17.85)$ & $07(29.16)$ & $08(36.36)$ & $20(27.02)$ \\
\hline B & $12(42.85)$ & $07(29.16)$ & $10(45.45)$ & $29(39.18)$ \\
\hline O & $10(35.71)$ & $05(20.83)$ & $02(09.09)$ & $17(22.97)$ \\
\hline AB & $01(03.57)$ & $05(20.83)$ & $02(09.09)$ & $08(10.81)$ \\
\hline Total & $28(100)(37.83)$ & $24(100)(32.43)$ & $22(100)(29.72)$ & $74(100)$ \\
\hline
\end{tabular}

Mild malaria cases were 10 in 'O' blood group while 18 in other blood groups. Severe malaria cases were 02 in 'O' blood group while 20 in others. Fisher exact test value was $0.045(\mathrm{P}<0.05)$. Thus ' $\mathrm{O}$ ' blood group had significantly less severe malaria cases and more mild malaria cases in comparison with other blood groups 


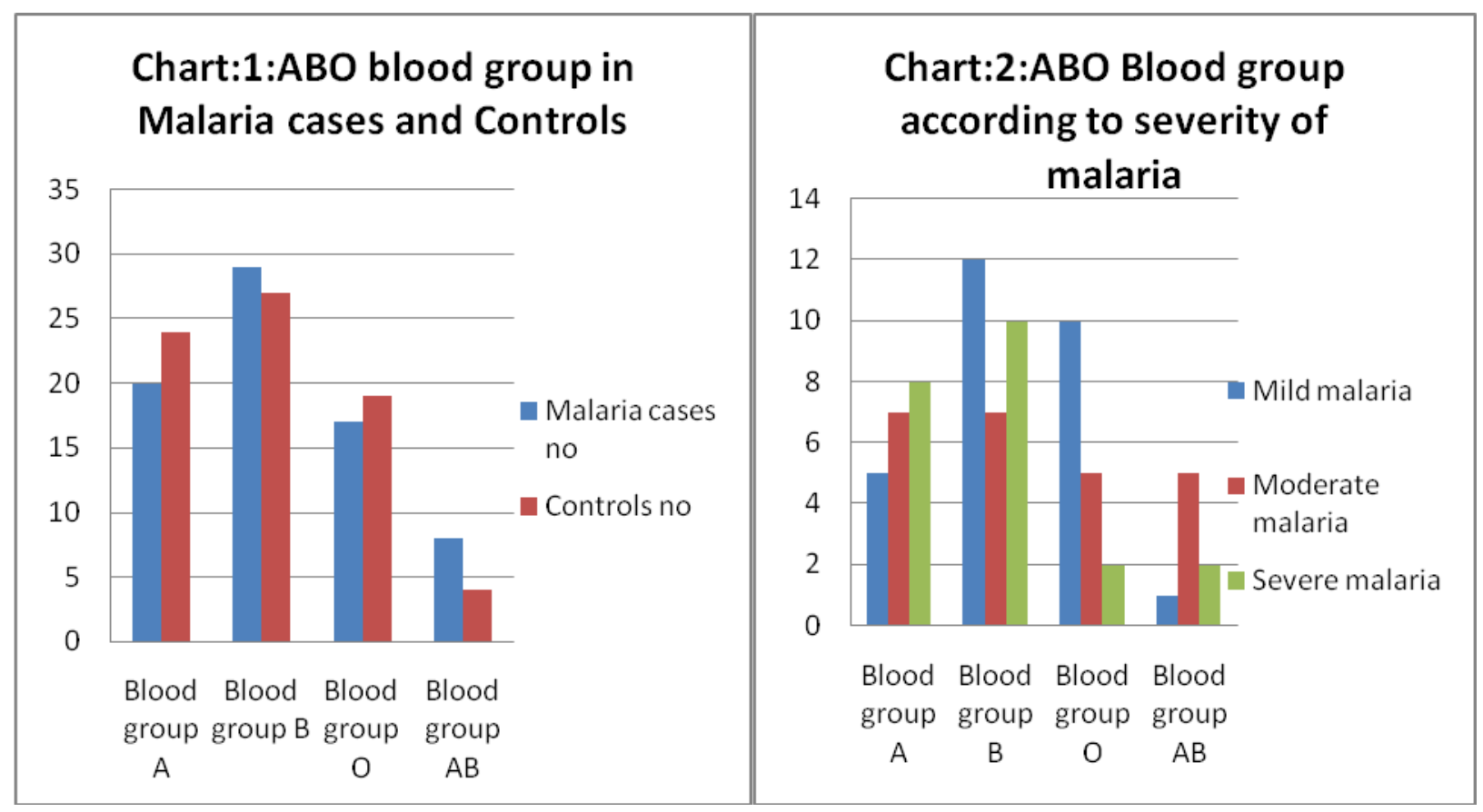

' $\mathrm{O}$ ' blood group had less number of severe malaria and more number of mild malaria cases in comparison with other blood groups. This was one of the significant finding of our study. Deepa et al., (2011) had similar finding in their study and concluded that ' $\mathrm{O}$ ' group had an advantage over the other three blood groups in relation to severity of the diseases. Present study suggests that ' $\mathrm{O}$ ' blood group had protection to severe malaria. In our study only 2 out of 22 severe malaria patient had 'O' blood group. Again of total 17 ' $\mathrm{O}$ ' blood group patients in our study, severe 10 had P.vivax and 1 had P.falciparum and 6 had mixed infection. Previous studies had shown that ' $\mathrm{O}$ ' blood group is protected from severe malaria. Reason which is given that ' $\mathrm{O}$ ' blood group RBC forms weak and small rosettes in comparison with non -'O' blood group ('A', 'B' and 'AB') (Carlson J et al., 1992).

If ' $\mathrm{O}$ ' blood group had protective effect than a question may arisen that malaria endemic area should have more community population of ' $\mathrm{O}$ ' blood group, based on hypothesis that blood group are expression of genetic constitution. We found in our community ' $\mathrm{B}$ ' blood group commonest in control group as well as in malaria case group and ' $\mathrm{O}$ ' blood group was present in only $22.97 \%$ in cases and $25.67 \%$ in control. More extensive work may resolve this problem. Many of infectious disease may be related to $\mathrm{ABO}$ blood group levels and secretes genes (Anstee et al., 2010). 'O' blood group is protected from severe malaria however it is also described in literature that occurrence of severe diarrhea due to E.coli, Vibrio cholarae and Helicobacter pylori is associated with ' $\mathrm{O}$ ' blood group. The reason is ' $O$ ' blood group genetic makeup has inability to secrete water (C. Caroline Blackwell et al., 2002)

In present day context significance of human blood groups are becoming more clearer due to its molecular basis (Yamamoto F. et al., 1990).There seems to be reasonable hypothesis that survival from malaria has resulted in blood group polymorphism. In today era of population movement, their host factor protective or susceptibility of various blood polymorphism may given up new insight in this field which may help in our challenge to fight malaria and other infectious 
disease in front other than antimalarials and antibiotics.

In conclusion, no special predilection was noted in regards to blood group and malaria. Except 'O' blood group had less severe malaria cases.

\section{References}

Aditya K Panda, Santosh K Panda, Aditya N Sahu, Rina Tripathy, Balachandran Ravindran and Bidyut $\mathrm{K}$ Das. Association of ABO blood group with severe falciparum malaria in adults: case control study and meta-analysis. Malaria Journal 2011; 10(309): 1-8

Anstee David. J. The relationship between blood groups and disease. Bristol Institute for Transfusion Sciences, National Health Service (NHS) Blood and Transplant, Bristol, United Kingdom 2010 by The American Society of Hematology Submitted January 21, 2010; accepted February 18, 2010. Prepublished online as Blood First Edition paper, March 22, 2010: DOI 10.1182/blood-2010-01-261859.

Beiguelman B, Alves FP, Moura MM, Engracia V, Nunes AC, Heckmann MI, et al., The association of genetic markers and malaria infection in the Brazilian Western Amazonian Region. Mem Inst Oswaldo Cruz.2003; 98:455460.

Carlson J, Wahlgren M. Plasmodium falciparum erythrocyte rosetting is mediated by promiscuous lectin-like interactions. J Exp Med 1992; 176:1311- 1317.

Caroline Blackwell, C., Stephanie Dundas, Valerie S. James, Doris A. C. Mackenzie, Jan M. Braun. Blood Group and Susceptibility to Disease Caused by Escherichia coli O157 University of Edinburgh, Edinburgh, and 3Infectious
Disease, Unit, Monklands District General Hospital, Airdire, Scotland, The Journal of Infectious Diseases 2002;185:393-6.

Christine M. Cserti and Walter H. Dzik. The ABO blood group system and Plasmodium falciparum malaria. Blood 2007; 110(7): 2250-2259

Deepa, Vanamala A, Karuna R, Cecil R. ABO blood group and malaria related outcome. J vector borne Dis 48, March 2011:7-11.

Fischer PR, Boone P. Short report: severe malaria associated with blood group. Am J Trop Med Hyg. 1998; 58:122123.

Gayathri B.N, Harendra Kumar M.L, Gomathi.N, Jeevan Shetty, Reethesh R. P. Relationship between ABO blood groups and malaria with clinical outcome in rural area of South India. Global Journal of Medicine and Public Health $2013 ; 2(2): 1-7$

Rowe J. Alexandra, Ian G. Handel, Mahamadou A. Thera, Anne-Marie Deans Blood group $\mathrm{O}$ protects against severe Plasmodium falciparum malaria through the mechanism of reduced resetting, www.pnas.org_cgi_doi_10. 1073_pnas.0705390104, PNAS, October 30, 2007 vol. 104 no. 44, 17471-1747

Singh G, Urhekar AD, Singh R. A study on correlation of malaria infection with A,B,O, RH blood group system. J Parasitol Vector Biol 2015;7:67-73

Samuel K. Martin Louis H. Miller C.U. Hicks Aba David-West C. Ugbode Mary Deane Frequency of blood group antigens in Nigerian children with falciparum malaria. Transactions of The Royal Society of Tropical Medicine and Hygiene, Volume 73, Issue 2, 1 January 1979, Pages 216-218, https://doi.org/10.1016/00359203(79)90217-7 
Thakur A, Verma IC. Malaria and ABO blood groups. Indian J Malariol 1992; 29: 241-4.

World malaria report 2014, apps.who.int/iris/bitstream/10665/14485 2/2/9789241564830.

Xuan Zhang, Meifang Yang, Hong Zhao, Jianhua $\mathrm{Hu}$, and Lanjuan $\mathrm{Li}$ Relationship between Malaria and ABO
Blood Types in $\backslash$ East China. Hindawi BioMed Research International Volume 2017, Article ID 8163762, 3 pages https: //doi.org /10.1155/ 2017/8163762 Yamamoto F et al., 1990, Clausen H, White $\mathrm{T}$, et al., Molecular genetic basis of the histo-blood group ABO system. Nature (Lond). 1990; 345(6272):229-233.

\section{How to cite this article:}

Sangita Vasava, Sucheta Lakhani and Jitendra Lakhani. 2019. Is there a Relation between ABO Blood Group and Malaria? Int.J.Curr.Microbiol.App.Sci. 8(03): 1153-1161. doi: https://doi.org/10.20546/ijcmas.2019.803.137 